\title{
Effect of Diabetes on the Male Reproductive System-A Histomorphological Study
}

\author{
Sushma R. Kotian ${ }^{1} \quad$ Ashok Kumar $^{1} \quad$ Sanchari Basu Mallik ${ }^{2} \quad$ Nandini P. Bhat $^{1} \quad$ Anne D. Souza ${ }^{1}$ \\ Arvind Kumar Pandey ${ }^{1}$
}

1 Department of Anatomy, Kasturba Medical College Manipal, Manipal Academy of Higher Education, Manipal, Karnataka, India

2 Department of Pharmacology Manipal College of Pharmaceutical Sciences, Manipal Academy of Higher Education, Manipal, Karnataka, India

Address for correspondence Arvind Kumar Pandey, PhD, Department of Anatomy, Kasturba Medical College Manipal, Manipal Academy of Higher Education, Manipal-576104, India

(e-mail: arvind.pandey@manipal.edu).

J Morphol Sci 2019;36:17-23.

\begin{abstract}
Keywords

- diabetes mellitus

- testis

- epididymis

- streptozotocin (STZ)

- male infertility

Introduction Type 1 diabetes is an autoimmune disorder characterized by lack of insulin production by the $\beta$ cells of the pancreas. This lack of insulin causes a variety of systemic effects on the metabolism of the body, one of which is reproductive dysfunction. The present study investigates the effects of diabetes on the male reproductive system of streptozotocin (STZ)-induced diabetic rats.

Material and Methods A total of 18 adult male Wistar rats weighing $\sim$ between 250 and $300 \mathrm{~g}$ were included in the present study. The animals were divided into normal and diabetic groups. The diabetic group was further subdivided into 2 subgroups with durations of 24 and 48 days. A single dose of STZ ( $40 \mathrm{mg} / \mathrm{kg}$ body weight) was administrated intraperitoneally to the animals of the diabetic group. After the planned duration, the testes and epididymides were dissected, and their gross weight was measured. The tissues were then processed for histological study.

Results The gross weight of the testes and epididymides in diabetic rats at 24 and 48 days showed a decrease in comparison to the control. ( $p<0.01$ for testes and epididymides).

Diabetic animals presented a significant decrease in the diameter of the seminiferous tubules compared with the control group $(p<0.01)$. The epididymides in the diabetic groups showed a considerable reduction in the tubular surface area compared with the control group $(p<0.01)$. There was also a reduction in the mean diameter, which was measured using the maximum and minimum diameter of the tubules $(p<0.01)$. Conclusion The present study is an insight into the adverse effects that diabetes can have on the tissue structure of the testes, of the epididymides, and ultimately on the process of spermatogenesis.
\end{abstract}

\section{Introduction}

Diabetes mellitus (DM) is characterized by an increased blood glucose level (hyperglycemia). The worldwide prevalence of DM was of $\sim 2.8 \%$ in 2000 , and it has been projected to extend to $4.4 \%$ in 2030 . The total figure of individuals with DM is estimated to escalate and double by 2030. Moreover, the occurrence of DM is higher in men than in women. ${ }^{1}$

Persistent hyperglycemia for long durations can lead to several problems, such as diabetic neuropathy, nephropathy, retinopathy, male impotence, and cardiovascular disease. ${ }^{2}$ received

December 12, 2018

accepted

February 2, 2019
DOI https://doi.org/

10.1055/s-0039-1683405. ISSN 2177-0298.
Copyright (c) 2019 by Thieme Revinter

Publicações Ltda, Rio de Janeiro, Brazil
License terms

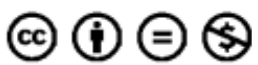


Although DM is responsible for the aforementioned problems, its effect on male sterility based on impotence, retrograde ejaculation, and hypogonadism is not widely understood.

The suggestion that DM has unfavorable effects on male fertility has long been contentious. ${ }^{3}$ Nevertheless, current studies have discovered that DM can affect the development of sperm and the production of androgens, which finally causes male infertility. ${ }^{4}$

Morphometric studies have shown a significant difference in the diameter of the seminiferous tubules in diabetic individuals and although a constant (20-25\%) total or subtotal block of spermatogenesis at spermatocytes stages 2 and 3 occur in small tubules of all animals. ${ }^{5}$

The streptozotocin (STZ)-induced diabetic rat model is one of the most extensively used models to study the effect of DM on fertility. Testicular dysfunction and degeneration were observed under conditions of experimentally induced DM in animal prototypes. ${ }^{6,7}$

The present study is, therefore, an attempt to investigate the effects of DM on the male reproductive system of STZinduced diabetic rat models. It also aims to quantify, through histological analysis, the rate of degeneration observed in the testes and in the epididymides caused by DM.

\section{Materials and Methods}

\section{Experimental Animals}

A total of 18 healthy adult male Wistar albino rats of both genders, weighing between 150 and $200 \mathrm{~g}$, housed under standard environmental conditions of temperature and humidity $\left(25 \pm 0.5^{\circ} \mathrm{C}\right)$ and 12 hour light/dark cycle were used for the present study. The animals were fed with standard pellet diet and water ad libitum. The experimental study was performed in the central animal house after obtaining the approval from the Institutional animal ethics committee (Ref. number: IAEC/KMC/49/2013).

The rats were randomly divided into 3 groups: control group ( $n=6)$, 28-days diabetic group $(n=6)$, and 46-days diabetic group $(n=6)$.

\section{Induction of Diabetes by Streptozotocin}

After 7 days of acclimatization, Wistar rats were used for the induction of DM. ${ }^{6}$ After overnight fasting, the rats were injected with a single intraperitoneal dose of streptozotocin (STZ) $(40 \mathrm{mg} / \mathrm{kg}$ ). Drinking water was added with glucose (5\%) to overcome the STZ-induced hyperglycemia. Fasting blood glucose levels were measured using an Accu-Chek Active glucometer with glucose oxidase-peroxide reactive strips (Roche Diagnostics India Pvt. Ltd., Mumbai, India). Animals with fasting blood glucose levels $>250 \mathrm{mg} / \mathrm{dl}$ were included in the present study.

On the confirmation of DM, the animals were observed for the planned duration, that is, 24 and 48 days, at the end of which the animals were sacrificed. The testes and the epididymides were dissected, and their gross weight was measured. The tissues were then fixed in $10 \%$ formalin for further studies.

\section{Microscopic Observations}

The fixed tissues were processed using paraffin embedding, and sections of $5 \mu \mathrm{m}$ thickness were acquired at $200 \mu \mathrm{m}$ recesses. The sections were subjected to hematoxylin and eosin (H\&E) staining to evaluate the testicular and epididymal morphology. The extent of tissue damage was analyzed, quantified and documented using microscopic images with the help of ImagePro Premier 9.1 (Media Cybernetics, Rockville, MD, USA).

\section{Histological Analysis}

The mean diameters of the seminiferous tubules in the testes and of the epididymal tubules were measured using an oculometer (Erma ocular micrometer, Japan) using a magnification of $10 \mathrm{x}$. It was performed in randomly selected 25 circular tubules in different slides of the same group and calculated with the following formula. ${ }^{8}$

Mean $=\sqrt{\text { smallest diameter }} \times$ larger diameter $\times$ magnification

Here, the smaller diameter was considered as the width of the tubules, and the large diameter as the length of the tubules.

Next, the histopathological changes in the testicular tissue were evaluated by the Johnsen testicular biopsy score system. It describes a new and rapid method for the registration of spermatogenesis in human testes. According to this system, each tubular section is given a score from 10 to 1 according to the incidence or to the nonappearance of the chief cell types organized in the order of development. Presence of spermatozoa scores 10 or 9; of spermatids scores 7 or 6 ; of spermatocytes scores 5 or 4 ; only spermatogonia scores 3; only Sertoli cells scores 2; and no cells scores 1 . A total of 50 cross-sectioned tubules in each group were evaluated systematically, and a score between 1 (very poor) and 10 (excellent) was given to each tubule according to the Johnsen criteria. ${ }^{9}$

\section{Statistical Analysis}

The results were expressed as mean \pm standard error of the mean (SEM). The data were analyzed using $R$ software ( $R$ Foundation for Statistical Computing, Vienna, Austria). Oneway analysis of variance (ANOVA) followed by the Dunnett post-hoc test was employed to compare the control and treated groups. The Tukey post hoc test was also used to compare between the different groups (control, 24-days, and 48-days). A $p$-value $\leq 0.05$ was considered statistically significant.

\section{Results}

\section{The Gross Size and Weight of the Testes and of the Epididymides}

The size of both the testes and the epididymides continued to show a major reduction on the $24^{\text {th }}$ and $48^{\text {th }}$ days in the diabetic groups compared with the control group (normal) (-Fig. 1).

The gross weight of the testes and of the epididymides in diabetic rats continued to reduce at 24 and 48 days in 

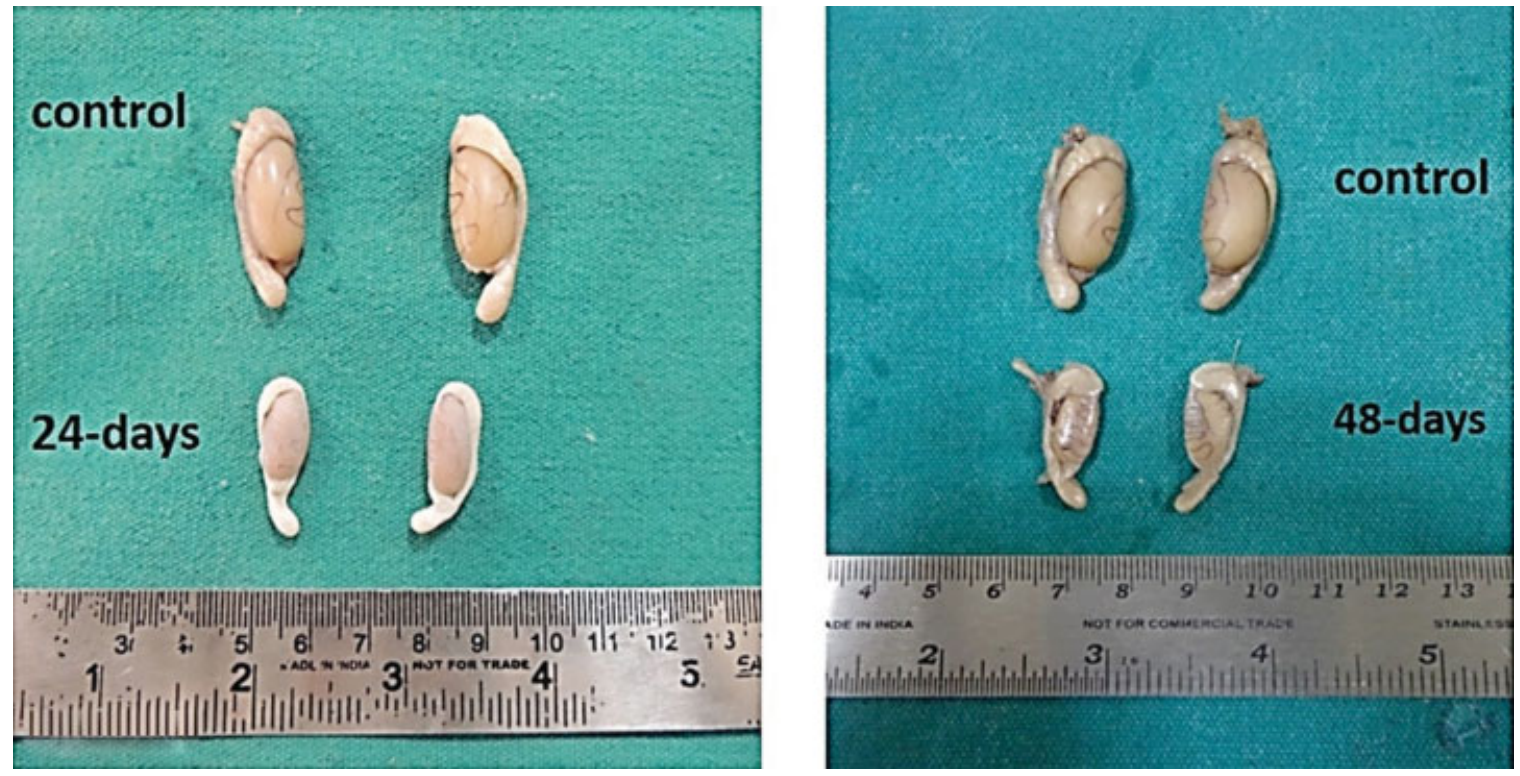

Fig. 1 Image showing the gross reduction in the size of the testes and of the epididymides in the 24-and 48-days diabetic groups in comparison with the control group.

comparison with the control group. The mean weight of the testes and of the epididymides was considerably lower in the rats of both 24- and 48-days groups compared with the control group (-Fig. 2), implying a significant weight reduction in the test groups ( $p<0.01$ for testes, $p<0.001$ for epididymis). Among the treated groups, the 48-days diabetic rats showed further reduction in the weight compared with the 24-days group. The findings were statistically significant ( $p<0.01$ versus the 24-days diabetic group). Although the epididymis showed a reduction, the findings were not statistically significant.

\section{Microscopic Observation}

\section{Histological Observation of the Testes}

The seminiferous tubules of the testes of the control group were healthy, showing normal testicular morphology, as well as all levels of spermatogenic cells. The Sertoli cells and the Leydig cells were viewed as normal (-Fig. 3a and b).
The diabetic animals presented a significant decrease in the diameter of the seminiferous tubules compared with the control group $(p<0.01)$. The structure of the tubules was disrupted. There was a considerable reduction in the number of the spermatogenic cells, that is the primary and secondary spermatocytes. Reduction in the number of spermatids was also perceived. There was a decrease in the Sertoli cells, as well as in the Leydig cells. The increased thickness of the basement membrane of the tubules was observed. Further, the spaces between the seminiferous tubules showed very less/scanty connective tissue (-Fig. $\mathbf{3 c}$, d, e, f).

The surface area of the most circular seminiferous tubules was reduced pointedly in the diabetic groups as opposed to the control group. The mean diameter that was calculated using the maximum length and breadth was decreased in the diabetic group. There was a steady decline in the testes biopsy score as calculated using the Johnsen criteria to quantify the nature of the tubules. ${ }^{9}$ All of the values were considerably reduced in the 48-days diabetic group in

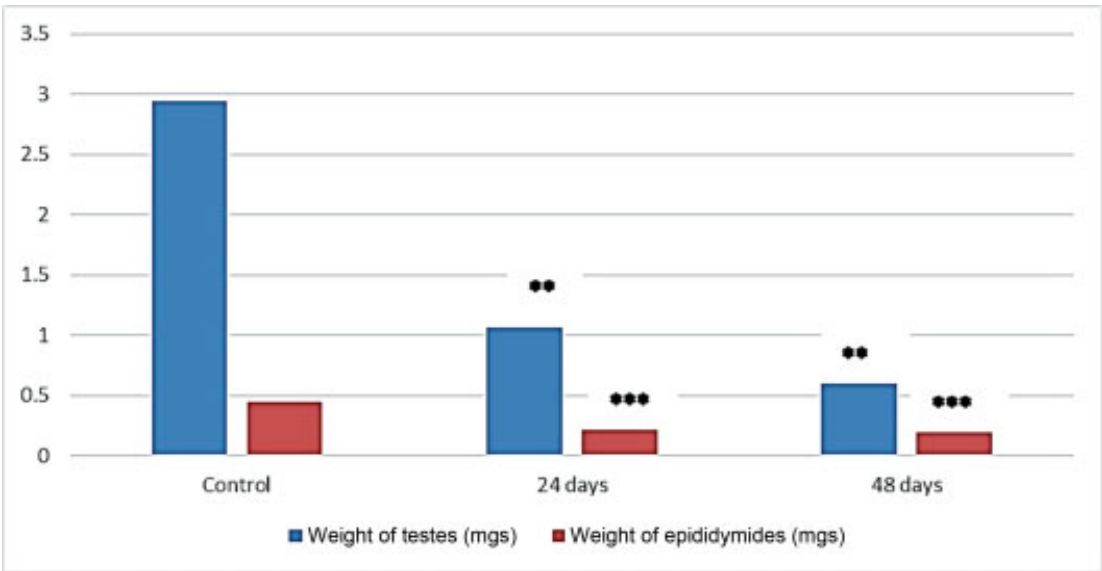

Fig. 2 Chart showing the comparison between the mean weight the testes and of the epididymides in the control and in the diabetic groups sacrificed at 24 and 48 days. ${ }^{* *} p$-value $<0.01 ;{ }^{* * *} p$-value $<0.001$ versuss control. 

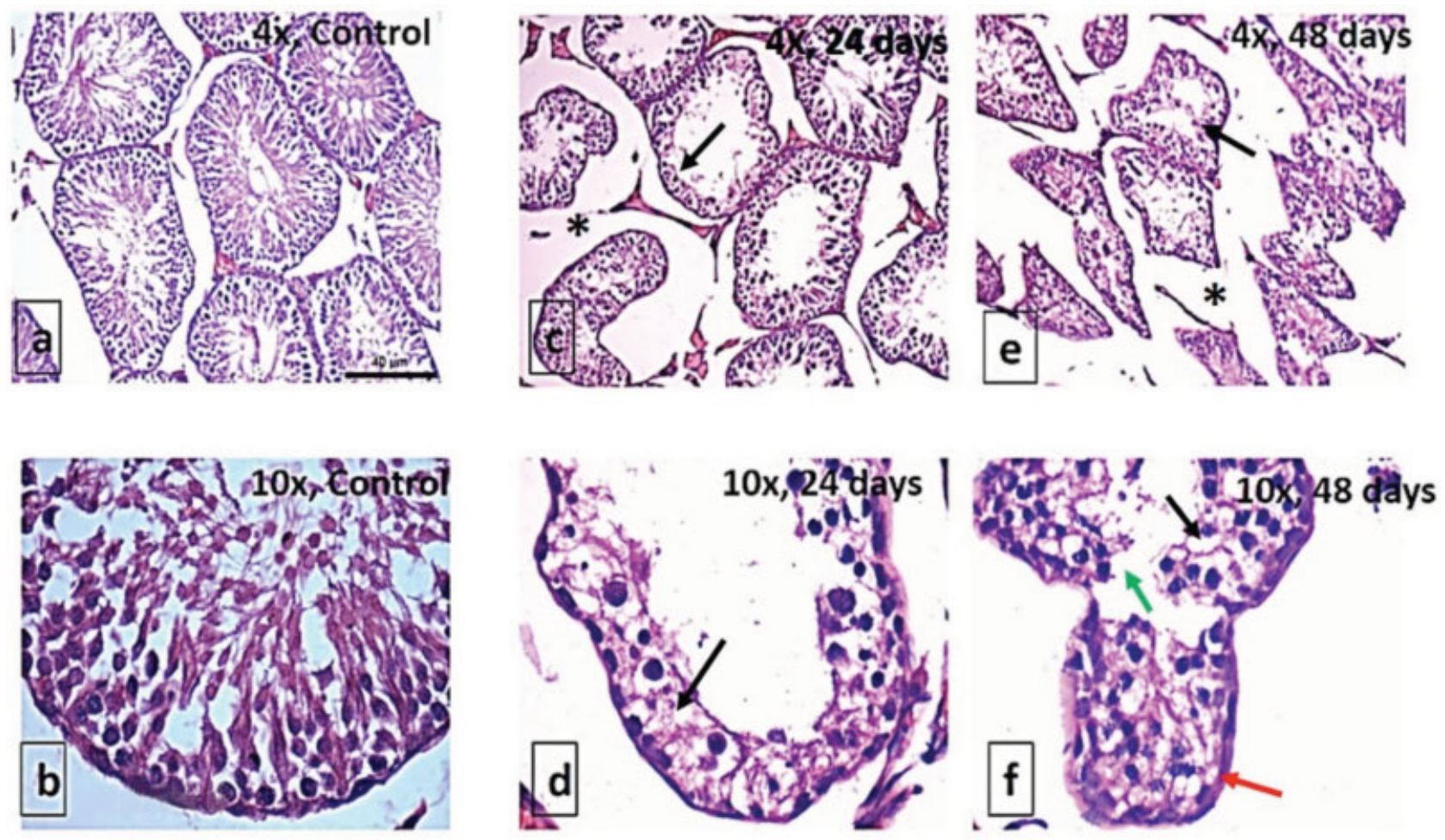

Fig. 3 Hematoxylin and eosin sections showing microscopic changes in the testicular tissue of the control and diabetic groups. (a) $4 x$ magnification shows the normal morphology of the seminiferous tubules, of the connective tissues, and of the Leydig cells. (b) $10 x$ magnification shows the normal morphology of single seminiferous tubules with normal Sertoli cells and all the layers of spermatogenic cells, from spermatogonia to spermatids. (c) $4 x$ magnification shows the degenerated seminiferous tubules (indicated by black arrow) and reduced levels of connective tissue and of Leydig cells (indicated by ${ }^{*}$ ). (d) 10x magnification of a single tubule with decreased spermatogenic cells and Sertoli cells (indicated by black arrow). (e) 4x magnification showing the decreased diameter of the seminiferous tubules (indicated by black arrow) and the degeneration of the connective tissue in the spaces between them (indicated by ${ }^{*}$ ). $(f) 10 x$ magnification of a single seminiferous tubule showing a thickened basement membrane (indicated by red arrow), very few spermatocytes (indicated by black arrow), and a narrow lumen (indicated by green arrow).

comparison with both the control and the 24-days diabetic group (-Table 1).

\section{Histological Observation of the Epididymis}

The microscopic structure of the epididymides in the control group showed normal morphology, the typical structure of the epididymal tubule, lining epithelium which is stratified columnar was noted, and abundant spermatozoa within the lumen were present (-Fig. $4 \mathbf{a}$ and $\mathbf{b}$ ).

The epididymides in the diabetic groups showed a considerable reduction in the tubular surface area compared with the control group $(p<0.01)$. There was also a reduction in the mean diameter, which was measured using the maximum and minimum diameter of the tubules $(p<0.01)$
(-Table 2). The lumen of the epididymis also showed a reduction in its dimensions, almost occluded lumen and completely devoid of spermatozoa ( $\mathbf{- F i g . ~} \mathbf{4 c}$, d, e, f). Clamping of the nuclei was observed, which was due to tubular shrinkage. The connective tissue between the tubules was sparse. The clamping of the nuclei showed an increase in the 48-days group in comparison both with the control and with the 24-days groups (-Table 2 ).

\section{Discussion}

Chronic hyperglycemia is a metabolic disease that threatens the world with multiple organ and system imbalances, including reproductive system dysfunction. Several results

Table 1 Comparing diameter, surface area and Johnsen score of seminiferous tubules of testes, between control and diabetic groups

\begin{tabular}{|l|l|l|l|}
\hline Parameter & Control & 24 days & 48 days \\
\hline Diameter of the tubules $(\mu \mathrm{m})$ & $73.83 \pm 12.95$ & $34.48 \pm 4.97^{* * *}$ & $20.82 \pm 6.41^{* * *}$ \\
\hline Surface area of the tubules $\left(\mu \mathrm{m}^{2}\right)$ & $449.22 \pm 150.69$ & $115.98 \pm 22.41^{* * *}$ & $70.39 \pm 40.41^{* * *}$ \\
\hline Johnsen score & $9.32 \pm 0.81$ & $4.06 \pm 0.91^{* * *}$ & $3.12 \pm 0.6^{* * *}$ \\
\hline
\end{tabular}

*** $p$-value $<0.001$ versus control. 

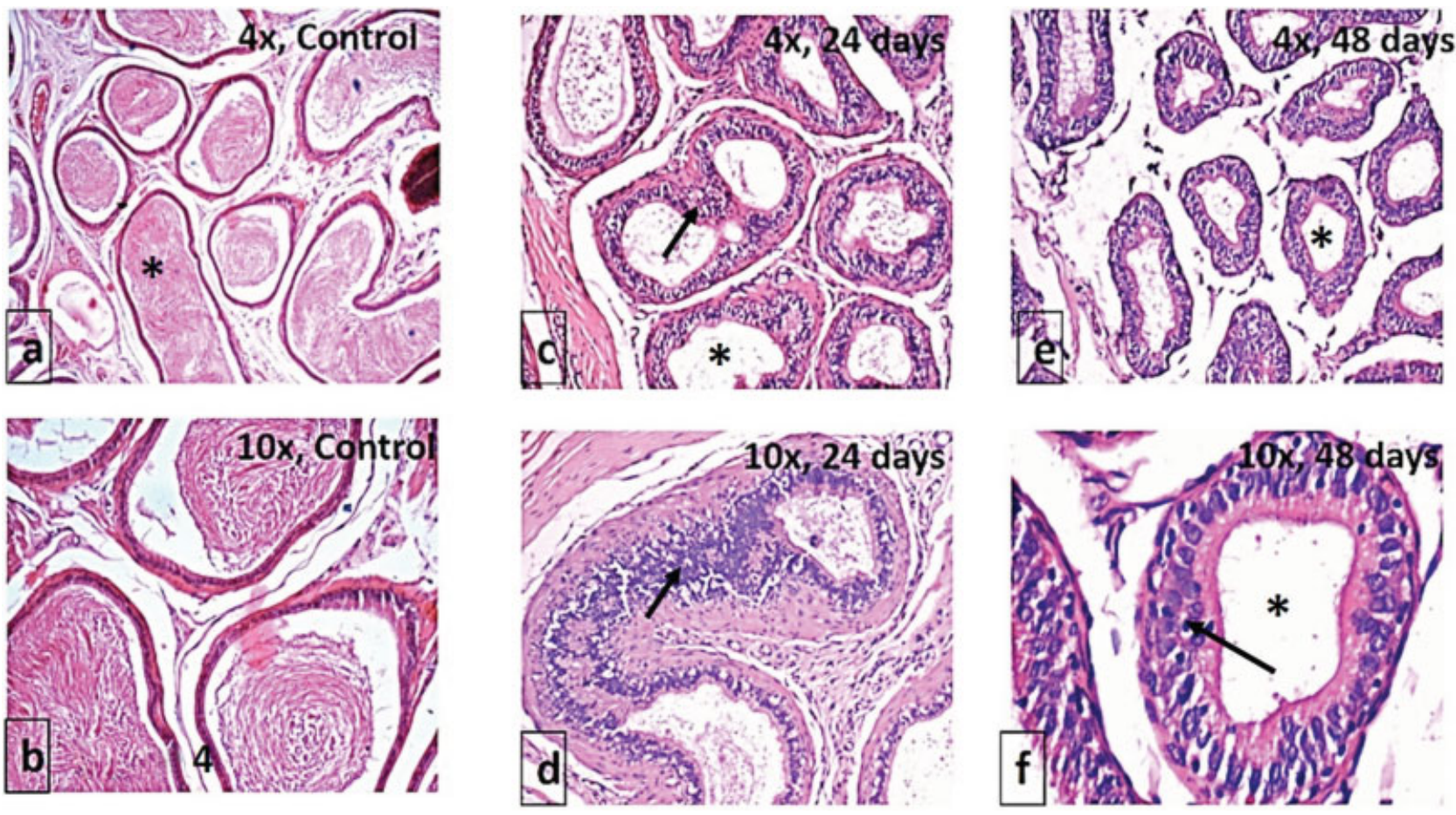

Fig. 4 Hematoxylin and eosin sections showing microscopic changes in the epididymides of the control and diabetic groups. (a) 4x magnification showing the normal morphology of the epididymides, and the lumen filled with abundant spermatozoa (indicated by ${ }^{*}$ ). (b) $10 x$ magnification showing normal lining epithelium and smooth muscle layer surrounding it. (c) 4x magnification of the 24-days diabetic group, note the shrinkage of the epididymal tubule; clamping of nuclei in the lining epithelium (indicated by black arrow) and reduced spermatozoa in the lumen (indicated by ${ }^{*}$ ). (d) 10x magnification of the seminiferous tubule of 24-days diabetic group showing nuclei clamping (indicated by black arrow), which almost occludes the lumen. (e) $4 x$ magnification of the 48-days diabetic group, showing the tubular diameter reduced, as well as the lumen reduced in size with no spermatozoa (indicated by ${ }^{*}$ ), connective tissue lost between the tubules, and the muscle layer diminished. (f) 40x magnification shows a single epididymal tubule of the 48-days diabetic group reduced in diameter with clamping of nuclei (indicated by black arrow), and the lumen completely devoid of spermatozoa (indicated by *).

showed that sexual behavior and reproductive tract functions are markedly affected by DM, which can lead to reduced fertility. Diabetic testicular damage may be brief or lasting, depending on the degree and on the duration of the disease. ${ }^{10}$

The present study showed that STZ-induced DM and increased blood glucose causes extensive histological changes in the testes and in the epididymides of rats.

In a study conducted by Guneli et al on STZ-induced diabetic rats, it was observed DM causes the reduction of spermatogenic cells, decreases the diameter of the seminiferous tubule, and increases the thickening of the basement membrane. It was perceived that the degeneration was caused by cell apoptosis as terminal deoxynucleotidyl transferase dUTP nick end labeling (TUNEL) positive cells were suggestively more in the test group. ${ }^{11}$ Ballester et al showed that the testicular sperm count, motility and testicular weight was pointedly reduced in diabetic rats. These changes were attributed to a significant reduction in the sex hormones (luteinizing hormone [LH] and follicle stimulating hormone $[\mathrm{FSH}]$ ) secreted by the pituitary gland in diabetic rats, which led to a decrease in the testosterone levels. ${ }^{5}$

In the present study, the gross weight of the testes and of the epididymides was considerably reduced in the test group. The diameter of the seminiferous tubule and the number of spermatogenic cells also showed a marked reduction in the rats of the diabetic groups compared with the control group.

Similar studies were conducted in the past by authors who concluded that the effects of DM on spermatogenesis

Table 2 Comparing diameter and surface area of tubules of the epididymides between control and diabetic groups sacrificed at 24 and 48 days

\begin{tabular}{|l|l|l|l|}
\hline Parameter & Control & 24 days & 48 days \\
\hline Mean diameter $(\mu \mathrm{m})$ & $1002.08 \pm 260.19$ & $701.24 \pm 132.57^{* * *}$ & $421.16 \pm 69.41^{* * *}$ \\
\hline Mean surface area $\left(\mu \mathrm{m}^{2}\right)$ & $1857.58 \pm 291.120$ & $831.47 \pm 160.11^{* * *}$ & $474 \pm 64.04^{* * *}$ \\
\hline Clamping of nuclei $(\mu \mathrm{m})$ & $215.75 \pm 63.76$ & $294.07 \pm 83.91^{* *}$ & $305.30 \pm 180.24^{* *}$ \\
\hline
\end{tabular}

${ }^{* * *} p$-value $<0.001$.

${ }^{* *} p$-value $<0.01$ versus control. 
could be identified as a lessening in the testicular diameter. Histological observation of calcification and sloughing of germ cells were also unquestionably seen. ${ }^{12,13}$ In the present study, the degeneration of the testes as calculated using the Johnsen testicular degeneration method further confirmed infertility in the diabetic groups.

Diabetes mellitus increases the thickness of the basal lamina of the tubules, which supplements the reduction of the total size of the tubular diameter of the epididymal tubules. $^{2}$ Likewise, the histological studies performed in the present scenario discovered a substantial increase in the thickness of the basal lamina, a reduction in the size of the tubules and in the lumen of the epididymal segments. Due to tubular shrinkage, the principal cells were packed tightly, showing clamping of the nuclei on one edge of the tubule. We have also measured the thickness of the clamping of the nuclei, which was seen to almost completely occlude the lumen. This finding was not documented previously in the literature.

Based on the studies conducted in the past, oxidative stress mechanisms were understood to contribute entirely or partially to the direction of the development of gonadal degeneration, utterly bereft of spermatozoa in STZ-induced diabetes in animal prototypes. ${ }^{14}$ Diabetes-induced weight loss of reproductive organs in males has also been reported to be caused by oxidative stress leading to the atrophy of the sex organs. ${ }^{15}$ The present study also showed a consistently significant reduction in the weights of the testes and of the epididymides. Numerous epididymal tubules were noted to be completely lacking in spermatozoa.

Hyperglycemia in diabetes increases the level of reactive oxygen species (ROS), which leads to DNA damage in the testes and, therefore, to a major reduction in the sperm motility, count, and viability. ${ }^{16}$ Diabetes mellitus also results in an increase in the levels of testicular malondialdehyde (MDA), a product of lipid peroxidation, and a reduction in the antioxidant levels, such as in the activity of superoxide dismutase (SOD), which leads to oxidative injury. ${ }^{17}$ An obvious increase in the MDA levels in diabetic rats has also been reported experimentally. ${ }^{17}$

Diabetes mellitus is also accompanied by the overexpression of inducible nitric oxide synthase (iNOS) and of nuclear factor kappa-light-chain-enhancer of activated B cells p-65 (NF-KB-p65), with a simultaneous upsurge in the testicular nitric oxide (NO) levels. The NO further produces reproductive dysfunction by causing testicular injuries that result in testicular atrophy and apoptosis. ${ }^{18,19}$

Aromatase, a member of the cytochrome P450 family, plays a vital role in the process of development and reproduction. ${ }^{20}$ It has been reported that the expression of aromatase markedly decreases in the testicular tissues of diabetic rats. ${ }^{21}$ Therefore, the reduced aromatase levels might be one of the critical mechanisms responsible for male reproduction dysfunctions in DM. ${ }^{16}$

The present study also endorses these molecular mechanisms as a possible explanation for the degenerative changes encountered in the testes and in the epididymides of diabetic rats.
In a study, 4 weeks after the STZ treatment, a significant increase in degenerated germ cells at various stages of development was observed. ${ }^{22}$ In the current scenario, degeneration was observed as beginning at 4 weeks, and it was more marked at 8 weeks. However, although STZ-induced DM in various animal studies has been demonstrated as a successful model for studying the manifestations of DM, it has been reported that high doses of STZ might induce damage in tissues besides the pancreas. ${ }^{10}$ In the past, authors have opined that the morphologic alterations observed in the testes of STZ-induced diabetic rats are not caused by a direct effect of the drug, but rather by DM. ${ }^{23}$ It remains uncertain if the damages are owing to DM or to STZ itself. Diabetic testicular dysfunction may be brief or lasting, depending on the degree and on the duration of the disease. ${ }^{12}$ However, the present study suggests that the long-term follow-up of 24 days and of 48 days of STZ-induced diabetic rat models showed significant changes in the histology of male reproductive organs. Moreover, it also showed a decrease in all types of spermatogenic cells in the seminiferous tubules, as well as absence of spermatozoa in the lumen of the epididymal tubules with clamping of the nuclei, which occluded the lumen of the tubules.

\section{Conclusion}

The present study gives an insight to the microscopic changes occurring in male reproductive organs, such as testes and epididymides in DM. We have made an attempt to quantify the changes and to provide reference data for future studies in the field. The present study contributes to the existing literature regarding the degenerative effects of STZ-induced DM on the male reproductive system.

Conflicts of Interest

The authors have no conflicts of interest to declare.

\section{References}

1 Wild S, Roglic G, Green A, Sicree R, King H. Global prevalence of diabetes: estimates for the year 2000 and projections for 2030. Diabetes Care 2004;27(05):1047-1053

2 Long L, Wang J, Lu X, et al. Protective effects of scutellarin on type II diabetes mellitus-induced testicular damages related to reactive oxygen species/Bcl-2/Bax and reactive oxygen species/microcirculation/staving pathway in diabetic rat. J Diabetes Res 2015; 2015:252530. Doi: 10.1155/2015/252530

3 Fedder J, Kaspersen MD, Brandslund I, Højgaard A. Retrograde ejaculation and sexual dysfunction in men with diabetes mellitus: a prospective, controlled study. Andrology 2013;1(04):602-606

4 Ramaswamy S, Weinbauer GF. Endocrine control of spermatogenesis: Role of FSH and LH/ testosterone. Spermatogenesis 2015;4 (02):e996025

5 Ballester J, Muñoz MC, Domínguez J, Rigau T, Guinovart JJ, Rodríguez-Gil JE. Insulin-dependent diabetes affects testicular function by FSH- and LH-linked mechanisms. J Androl 2004;25 (05):706-719

6 Maritim AC, Sanders RA, Watkins JB III. Diabetes, oxidative stress, and antioxidants: a review. J Biochem Mol Toxicol 2003;17(01):24-38

7 Shrilatha B, Muralidhara. Early oxidative stress in testis and epididymal sperm in streptozotocin-induced diabetic mice: its progression and genotoxic consequences. Reprod Toxicol 2007;23 (04):578-587 
8 Khaneshi F, Nasrolahi O, Azizi S, Nejati V. Sesame effects on testicular damage in streptozotocin-induced diabetes rats. Avicenna J Phytomed 2013;3(04):347-355

9 Johnsen SG. Testicular biopsy score count-a method for registration of spermatogenesis in human testes: normal values and results in 335 hypogonadal males. Hormones 1970;1(01):2-25

10 La Vignera S, Condorelli R, Vicari E, D’Agata R, Calogero AE. Diabetes mellitus and sperm parameters. J Androl 2012;33(02):145-153

11 Guneli E, Tugyan K, Ozturk H, Gumustekin M, Cilaker S, Uysal N. Effect of melatonin on testicular damage in streptozotocininduced diabetes rats. Eur Surg Res 2008;40(04):354-360

12 Altay B, Cetinkalp S, Doganavşargil B, Hekimgil M, Semerci B. Streptozotocin-induced diabetic effects on spermatogenesis with proliferative cell nuclear antigen immunostaining of adult rat testis. Fertil Steril 2003;80(02, Suppl 2):828-831

13 Rohrbach DH, Martin GR. Structure of basement membrane in normal and diabetic tissue. Ann N Y Acad Sci 1982;401:203-211

14 Soudamani S, Yuvaraj S, Malini T, Balasubramanian K. Experimental diabetes has adverse effects on the differentiation of ventral prostate during sexual maturation of rats. Anat Rec A Discov Mol Cell Evol Biol 2005;287(02):1281-1289

15 Sancheti S, Bafna M, Seo S. Anti hyperglycemic, anti hyperlipidemic, and antioxidant effects of Chaenomelessinensis fruit extract in streptozotocin induced diabetic rats. Eur Food Res Technol 2010;231:415-421
16 Amaral S, Oliveira PJ, Ramalho-Santos J. Diabetes and the impairment of reproductive function: possible role of mitochondria and reactive oxygen species. Curr Diabetes Rev 2008;4(01): 46-54

17 Bauché F, Fouchard MH, Jégou B. Antioxidant system in rat testicular cells. FEBS Lett 1994;349(03):392-396

18 Kushwaha S, Jena GB. Telmisartan ameliorates germ cell toxicity in the STZ-induced diabetic rat: studies on possible molecular mechanisms. Mutat Res 2013;755(01):11-23

19 Singh VK, Lal B. Pro-steroidogenic and pro-spermatogenic actions of nitric oxide (NO) on the catfish, Clarias batrachus: An in vivo study. Gen Comp Endocrinol 2017;242:1-10

20 Rodriguez-Castelan J, Mendez-Tepepa M, Carrillo-Portillo Y, et al. Hypothyroidism reduces the size of ovarian follicles and promotes hypertrophy of periovarian fat with infiltration of macrophages in adult rabbits. BioMed Res Int 2017;2017:3795950

21 Burul-Bozkurt N, Pekiner C, Kelicen P. Diabetes alters aromatase enzyme levels in gonadal tissues of rats. Naunyn Schmiedebergs Arch Pharmacol 2010;382(01):33-41

22 Navarro-Casado L, Juncos-Tobarra MA, Cháfer-Rudilla $M$, de Onzoño LÍ, Blázquez-Cabrera JA, Miralles-García JM. Effect of experimental diabetes and STZ on male fertility capacity. Study in rats. J Androl 2010;31(06):584-592

23 Oksanen A. Testicular lesions of streptozotocin diabetic rats. Horm Res 1975;6(03):138-144 